\title{
CORRIGENDUM
}

doi:10.1038/nature11977

\section{Corrigendum: Structure-based prediction of protein-protein interactions on a genome-wide scale}

Qiangfeng Cliff Zhang, Donald Petrey, Lei Deng, Li Qiang, Yu Shi, Chan Aye Thu, Brygida Bisikirska, Celine Lefebvre, Domenico Accili, Tony Hunter, Tom Maniatis, Andrea Califano \& Barry Honig

Nature 490, 556-560 (2012); doi:10.1038/nature11503

In this Letter, one of the points shown in Fig. 2 and Supplementary Figs 8, 9 and Supplementary Table 4 reflects the presence of interactions that had been erroneously deposited from a previous publication ${ }^{1}$ into the IntAct database. We have now used the MINT database to retrieve these interactions, and Fig. 2 is corrected here (shown below as Fig. 1). The error in IntAct was corrected on 9 November 2012 in consultation with the original authors of the paper. We thank S. Michnick for bringing this to our attention. We also thank M. Maletta for pointing out that Supplementary Fig. 10C was mislabelled and erroneously indicated that NKX2-2 protein was not included in the experiment. See Supplementary Information to the original paper for corrected versions of Supplementary Figs 8-10C and Supplementary Table 4. These errors do not affect the results or conclusion of the paper, and have been corrected in the HTML and PDF of the original paper.

1. Taeassov, K. et al. An in vivo map of the yeast protein interactome. Science $\mathbf{3 2 0}$, $1465-1470$ (2008)

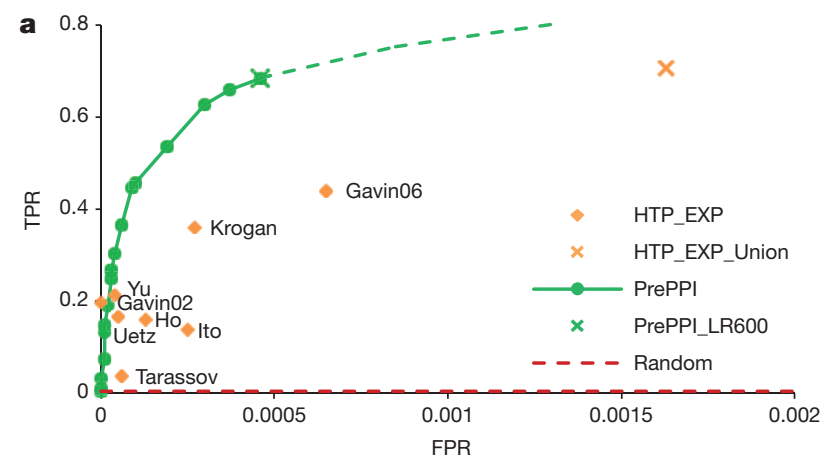

b

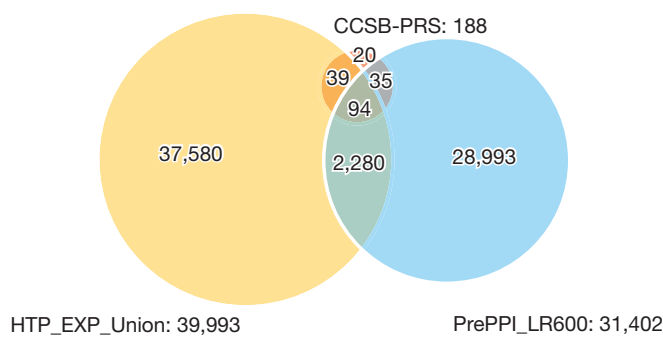

Figure $1 \mid$ This is the corrected Fig. 2. 